\title{
Mirror boxes and mirror mounts for photophysics beamline
}

\author{
PMEENAKSHI RAJA RAO ' , B NRAJASEKHAR ', NCDAS ', H A KHAN ${ }^{1}$, \\ S S BHATTACHARYA ', A S RAJA RAO ${ }^{2}$ and A P ROY ${ }^{\prime}$ \\ ' Spectroscopy Division, Bhabha Atomic Research Centre, Mumbai 400085 , \\ India \\ ${ }^{2}$ Centre For Advanced Technology, Indore 452 013, India \\ MS received 17 September 1996; revised 16 June 1997
}

\begin{abstract}
A medium resolution beamline, viz. photophysics beamline is being built at INDUS-I, a $450 \mathrm{Mev}$ synchrotron radiation source (SRS) at the Centre for Advanced Technology (CAT). Indore. To house the pre- and postfocusing toroidal mirrors and permit precision movements for steering the SRS beam up to the sample, two ultra-high vacuum (UHV) compatible mirror boxes and mirrors have been designed, fabricated and tested. Details of the setup, including UHV testing ( $P<10^{-9} \mathrm{mbar}$ ) and performance evaluation of the mirror mounts under UHV conditions, are discussed in this paper.
\end{abstract}

Keywords. Mirror box; mirror mount; photophysics beamline; residual gas spectrum; INDUS-1.

\section{Introduction}

INDUS-I is a $450 \mathrm{Mev}$ synchrotron radiation source (SRS) being constructed at the Centre for Advanced Technology, Indore (Ramamurthi 1992). The radiation emitted from the storage ring is useful for performing experiments in the soft X-ray to infrared region. A beamline, viz. photophysics beamline dedicated to experiments on photophysics at INDUS-I, is under fabrication. This beamline makes use of a toroidal mirror for focusing the light $[40 \operatorname{mrad}(H) \times 6 \operatorname{mrad}(V)]$ from the tangent point of INDUS-I onto the entrance slit of a one-metre Seya-Namioka type of monochromator and a second toroidal mirror for focusing the monochromatic light emerging from the exit slit of the monochromator onto the sample $(1 \mathrm{~mm} \times 1 \mathrm{~mm}$ spot size $)$ positioned at a distance of one metre from the centre of the focusing mirror. Detailed optical and mechanical layouts of the beamline formed the basis of the mirror box and mirror mount designs (Das \& Rajasekhar 1992; Meenakshi Raja Rao et al 1992). A side view of the photophysics beamline is shown in figure 1. Ultimate pressure in the beamline has to be maintained at $<10^{-9} \mathrm{mbar}^{*}$. In addition to

${ }^{*} 1 \mathrm{mbar}=10^{2} \mathrm{~Pa}$ 


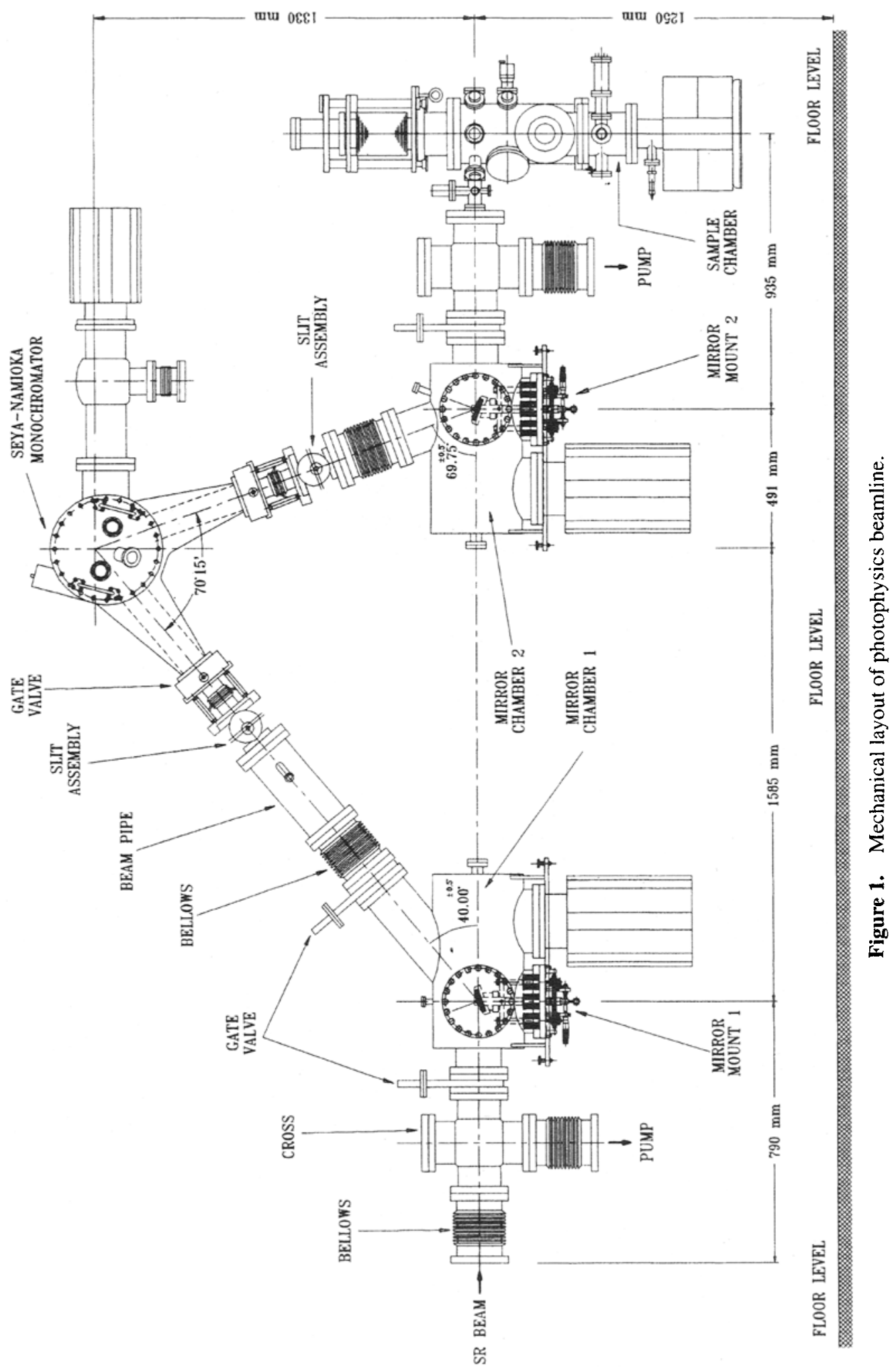


being the prime requirement for connecting the beamline to the storage ring which operates at a pressure of $<10^{-9} \mathrm{mbar}$ such ultra-high vacuum (UHV) environment also protects the surfaces of the optical components. Installation of the beamline to get maximum flux at the sample position requires precise and reproducible rotational and translational movements of the mirrors about the $X, Y$ and $Z$ axes under ultra-high vacuum conditions so as to steer the synchrotron radiation source (SRS) beam up to the sample. The fine movements required are achieved with the help of UHV compatible mirror mounts. Details of design, fabrication and testing of the mirror mounts and mirror chambers are described below.

\section{Description}

\subsection{Design considerations}

2.1a Mirror mounts: In case of mirror mounts for holding both pre- and post-mirrors, and adjusting their alignment and fine movements, the considerations are

1) Rotational and translational motions in three mutually perpendicular directions ( $X, Y$ and $Z$ ) should be independent. They should not disturb the prevailing UHV conditions of the beamline.

2) Accuracies of translational movements should be of the order of $1 \pm 0.5 \mathrm{~mm}$.

3) Accuracies of rotational movements should be of the order of $1 \pm 0.2$ milliradian.

If the linear and angular positions are not maintained within the accuracies mentioned above, the image at sample position will be shifted from the mean position by about $0.3 \mathrm{~mm}$ resulting in loss of flux of the order of $25 \%$.

2.1b Mirror boxes: The UHV mirror boxes needed several ports of various sizes not only to connect them to the beamline but also to connect mirror mounts, gauge heads etc. In addition, ports also were provided for handling the mirror mounts and viewing the beam spot on the mirror. Table 1 gives a brief description of the ports of the mirror boxes.

Table 1. Description of the ports of the mirror boxes.

\begin{tabular}{lcl}
\hline Flange & Quantity & \multicolumn{1}{c}{ Description } \\
\hline $203 \mathrm{CF}$ & 1 & Mirror mount (Port 1) \\
$152 \mathrm{CF}$ & 5 & Beam entry (Port 2) \\
& & Beam exit (Port 3) \\
& & Manual alignment (Ports 4 \& 5) \\
$35 \mathrm{CF}$ & 3 & Sputter ion pump (Port 6) \\
& & BA Gauge (Port 7) \\
$16 \mathrm{CF}$ & 1 & Roughing pump (Port 8) \\
& & Pirani-Penning Gauge (Port 9) \\
\hline
\end{tabular}




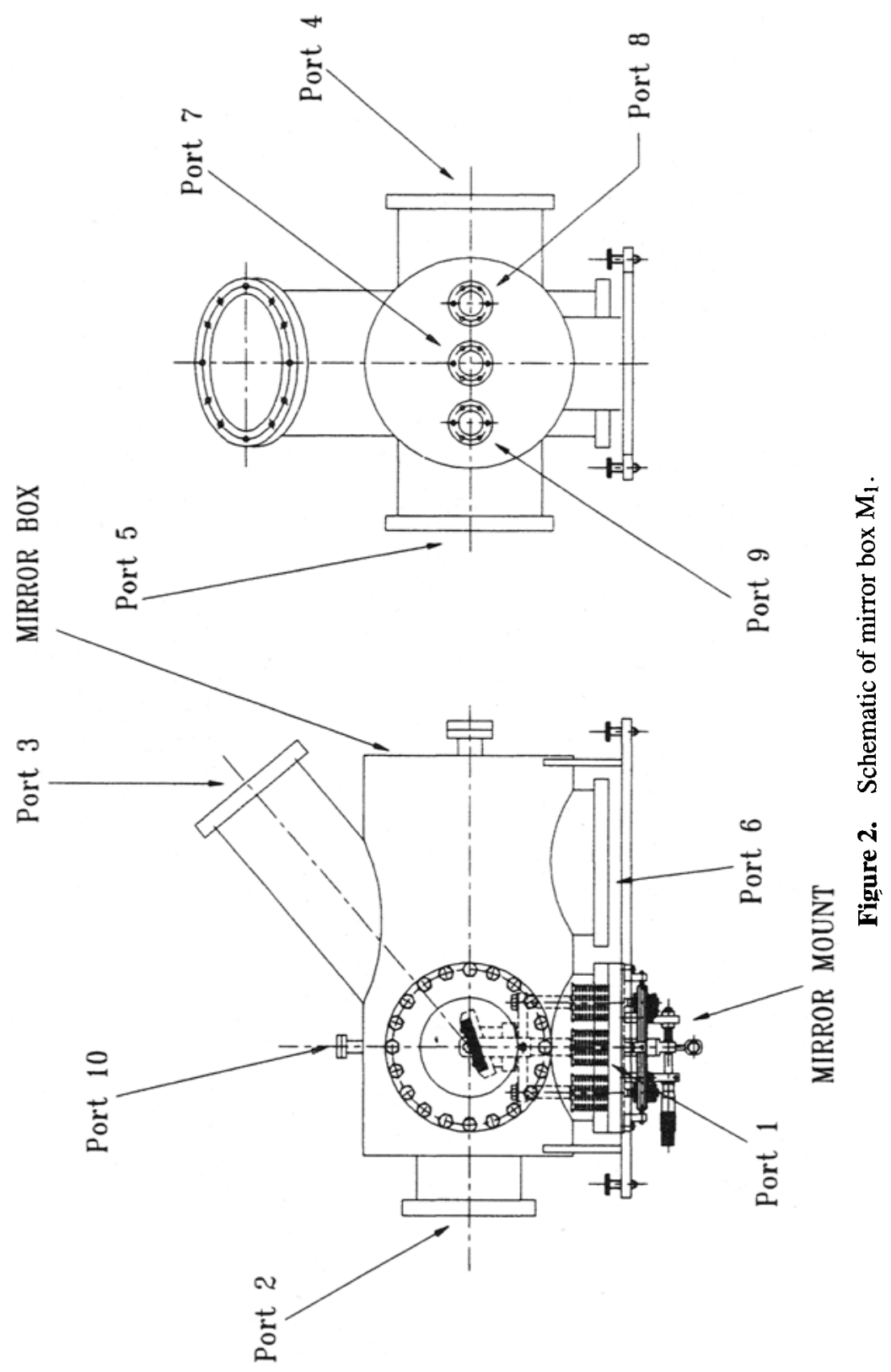




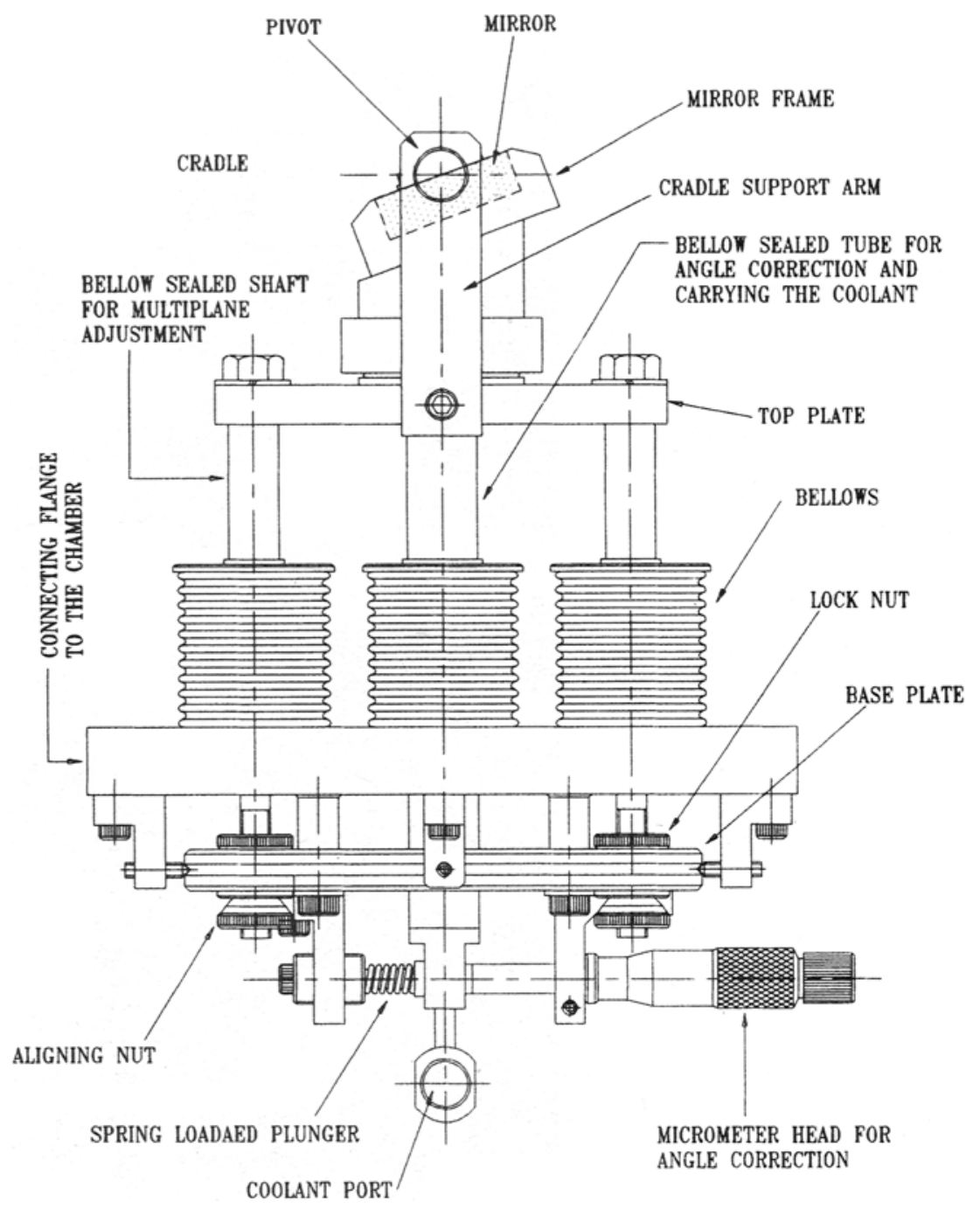

Figure 3. Schematic of mirror mount $\mathrm{M}_{1}$.

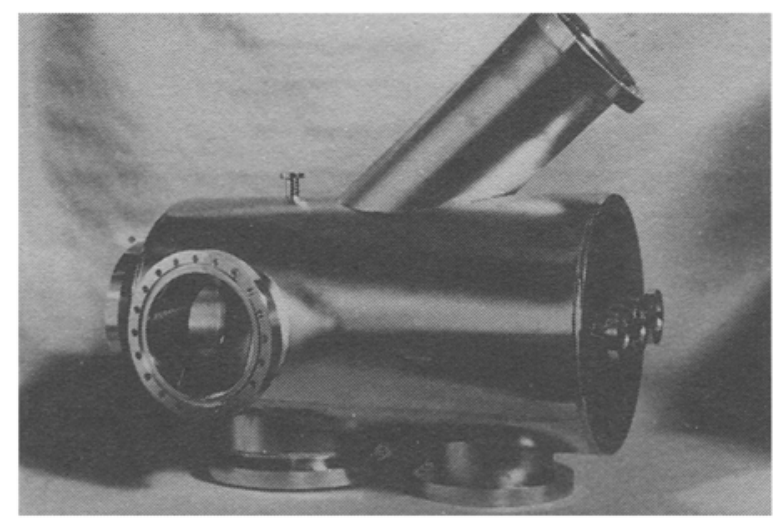

Figure 4. Photograph of mirror box. 


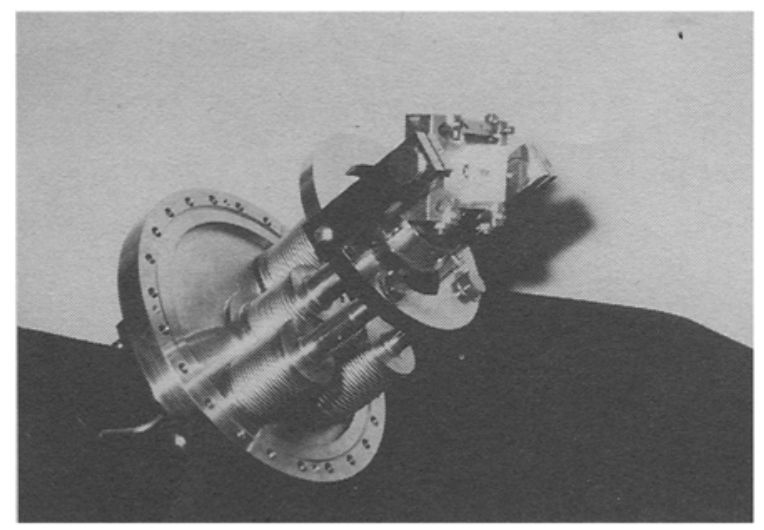

Figure 5. Photograph of mirror mount.

\section{Design details}

Based on the design considerations mentioned above and on the guidelines from the beamlines of the Ultraviolet Synchrotron Orbital Radiation Facility (UVSOR), Japan (Sakai $e t$ al 1987), mirror chambers and mirror mounts were designed and fabricated. The two mirror boxes are mirror images of each other with a few minor differences depending on the entry and exit of light (Ramamurthi 1992; Meenakshi Raja Rao et al 1992, 1995). Schematics of the mirror boxes and mirror mounts are shown in figures 2 and 3 respectively. Figures 4 and 5 show photographs of the mirror mount and mirror chamber respectively. The mirror is enclosed in a frame provided with adjustable screws on the mirror mount. This arrangement facilitates the rotation of the mirror around an axis which is perpendicular to the plane of the mirror. The mirror frame is mounted on a copper block having an angle equal to the mirror tilt. The mirror frame has an arrangement for locating the mirror accurately by using fine pitch positioning screws. Two pivot joints provided on the frame impart the rotation necessary for angle correction. Rotation of the mirror is controlled from outside by a bellows-sealed mechanism using a micrometer head and a spring loaded plunger. The tube which imparts this rotation also acts as a carrier for coolant to the mirror frame. Multiplane adjustment of the mirror is provided by using three long bellows-sealed shafts located in a right-angled triangular geometry. The mirror mount is welded to a $203 \mathrm{CF}$ flange and this flange is connected to port 1 of the mirror box.

\section{Materials and fabrication}

SS 304L has been used for fabrication of most of the components of the mirror mounts and mirror boxes. The bellows and coolant channel used are made of SS 316. The supporting block of the mirror holder is made of copper for better thermal conductivity. The chambers and mounts are TIG-welded from inside, cleaned, electropolished, and degassed in a vacuum furnace at a temperature of $800^{\circ} \mathrm{C}$ for $8 \mathrm{~h}$. This procedure reduced degassing thereby saving time taken for achieving ultimate pressure. 


\section{Testing setup}

The mirror mounts were connected to the mirror chamber after degassing all the components and the mirror box in a vacuum furnace. A $2701 / \mathrm{s}$ sputter ion pump was used to achieve the ultimate pressure. A pirani gauge was used to measure pressure up to $10^{-3} \mathrm{mbar}$. A Bayard-Alpert nude ionisation gauge (model IG5M of M/S Edwards, UK) was used to measure pressure from $10^{-4}$ mbar to $10^{-9}$ mbar and a penning gauge and a leak detector were connected to the chamber with the help of a " $T$ " through a turbomolecular pumping station (Model Turbopac 5150 of M/S Alcatel, France). The rest of the ports of the chambers were blanked off. Two sight glasses were provided for the entry and exit of collimated laser light from $\mathrm{He}-\mathrm{Ne}$ Laser used for testing the mirror mount mechanism. All gauges, gauge control units, pumps and their accessories were provided power through the regulated power supply. Heating of the chamber, mount, ion pump etc. for degassing purposes was carried out using different heating elements operated through temperature controllers. All the vacuum sealings were done by metal gaskets made of OFHC copper. The experimental setup showing different instruments used for leak detection and evaluation is shown in figures 6 and 7.

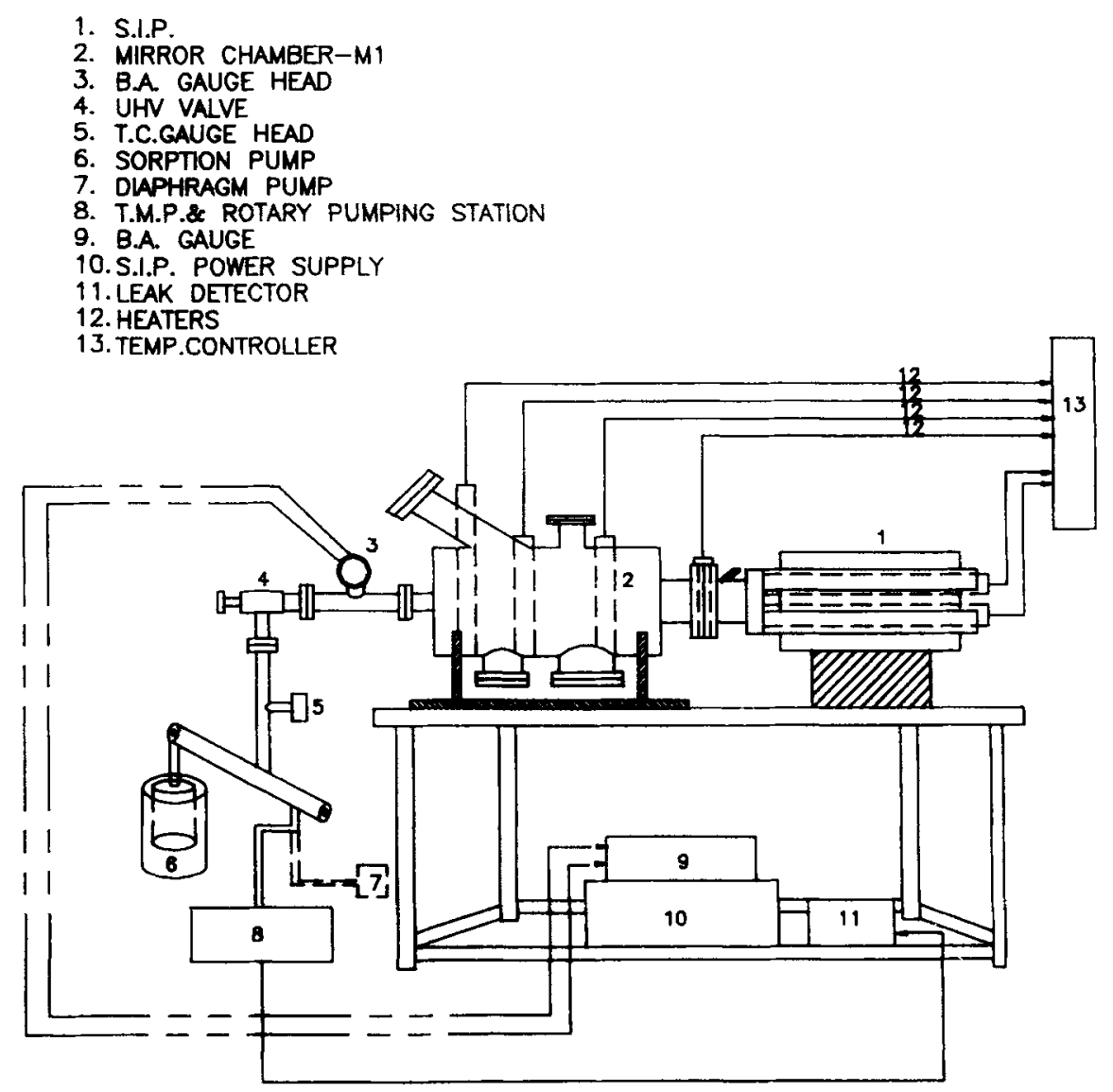

Figure 6. Side view of the testing setup (drawing is not to scale). 
16. SORPTION PUMP

17. MASS ANALYSER HEAD

18. R.F. HEAD

19. RECORDER

20. OMS CONTROLIER

21. PERSONAL COMPUTOR

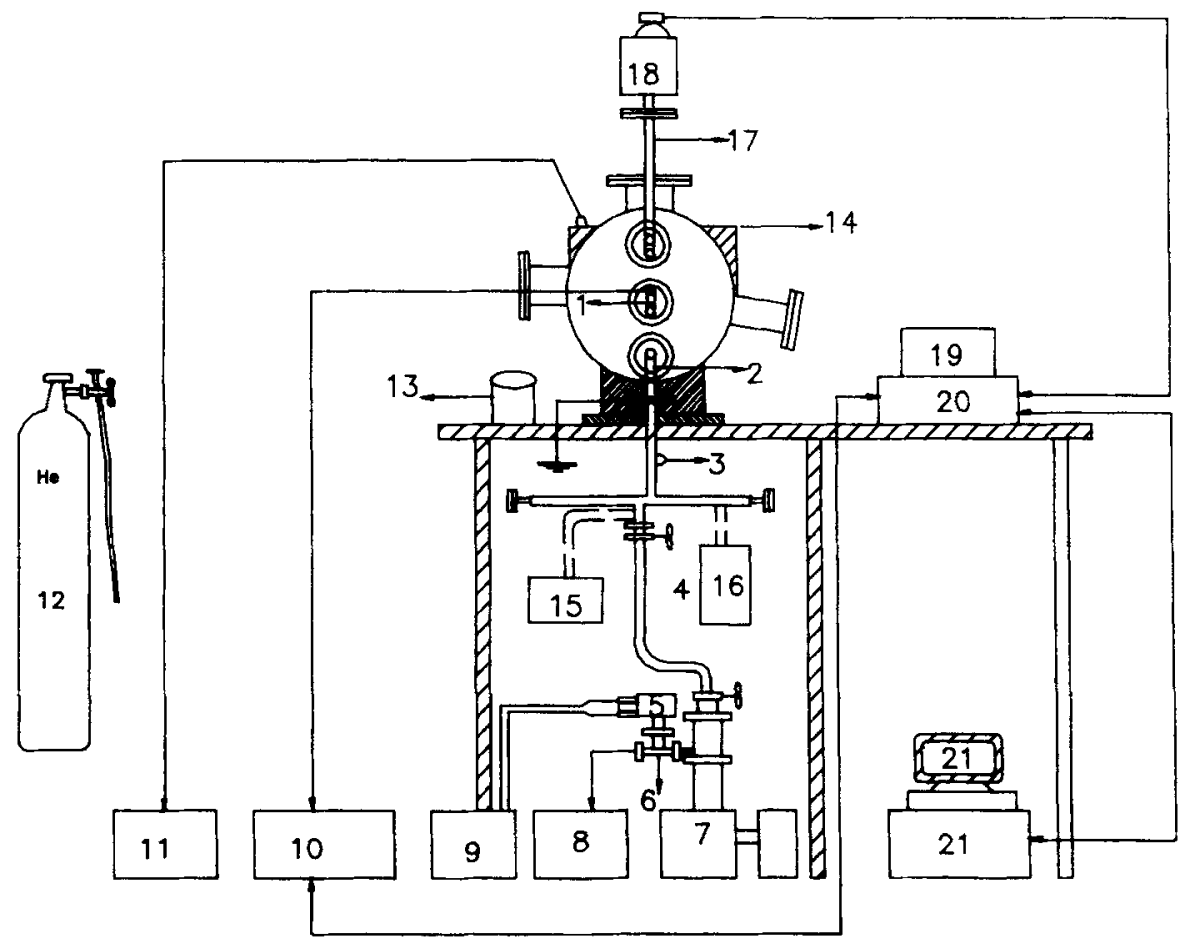

Figure 7. Front view of the testing setup (drawing is not to scale).

\section{Testing procedure}

To start with, the mirror chamber was leak-tested for welding and gasket leaks of the order of $10^{-10} \mathrm{std} . c \mathrm{c} / \mathrm{s}^{*}$. A pressure of $1.0 \times 10^{-8} \mathrm{mbar}$ was achieved with the help of a turbomolecular pump and a sputter ion pump without baking the chamber or the sputter ion pump (unconditioned). The residual gas spectrum was recorded using a quadrupole mass analyzer to ascertain the various components of residual gases and their relative concentrations as shown in figure 8 . After baking the sputter ion pump and the chamber for about $8 \mathrm{~h}$ at $250^{\circ} \mathrm{C}$ and pumping the chamber with sputter ion pump, a pressure of $1.0 \times$ $10^{-9}$ mbar was achieved. The residual gas spectrum was recorded again at this pressure (figure 9). From figures 8 and 9 it can be seen that water vapour, $\mathrm{CO}_{2}, \mathrm{~N}_{2}$ and hydrocarbons, which were the major constituents before degassing, have been suppressed and that $\mathrm{H}_{2}$ is the only predominant gas component after baking. Thus hydrocarbon-free ultra-high

$\overline{{ }^{*} 0.987 \mathrm{std} . \mathrm{cc} / \mathrm{s}}=0.1 \mathrm{~Pa} \mathrm{~m}^{3} \mathrm{~s}^{-1}$ 


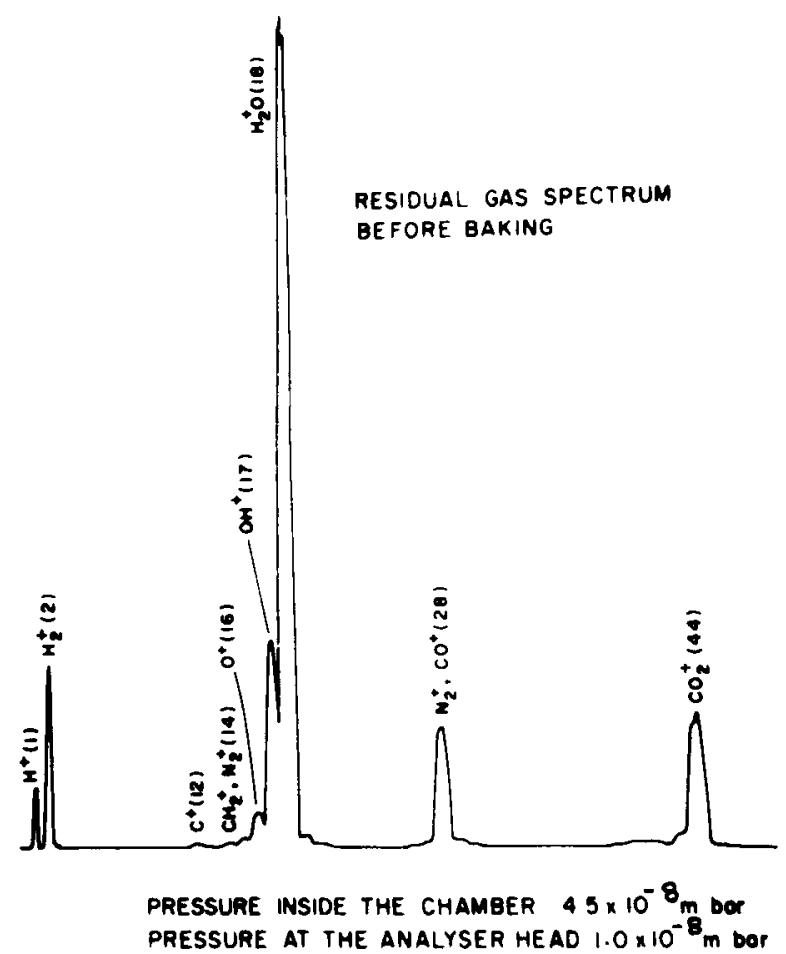

Figure 8. Residual gas spectrum before baking the mirror chamber.

vacuum essential for SRS was achieved. After achieving this ultimate pressure, the mirror mounts were tested for their performance under UHV conditions (i.e., reproducibility and minimum attainable translational and rotational displacement) using an $\mathrm{He}-\mathrm{Ne}$ laser. This test was performed by rotating the mirror about an axis which is perpendicular to the incident beam direction and measuring the image displacement on a screen placed at a distance of $1250 \mathrm{~mm}$ (corresponding to the distance between pole of the first mirror and entrance slit of the monochromator). The minimum rotation of the micrometer screw imparted a rotation of $0.0033^{\circ}$ to the mirror which resulted in an image displacement of $75 \mu$ at the entrance slit. This is adequate as entrance slit width is planned to be of the order of $100 \mu$. For the second mirror mount similar procedure was repeated and same result of $75 \mu$ is obtained as the minimum displacement of the image and is considered to be very good for a spot size of $1 \mathrm{~mm} \times 1 \mathrm{~mm}$ at the sample position.

\section{Conclusions}

Two mirror chambers and two mirror mounts for photophysics beamline were designed, fabricated and tested. The mirror mounts were tested for their performance at atmospheric pressure and at a pressure of $1.0 \times 10^{-9} \mathrm{mbar}$. The residual gas spectrum recorded at $1.0 \times 10^{-9}$ mbar indicated that $\mathrm{H}_{2}$ is the major constituent of the residual gases. The minimum displacement of the image at the entrance slit (for mirror $\mathbf{M}_{1}$ ) and at sample (for the mirror $\mathrm{M}_{2}$ ) is $75 \mu$. This value is adequate as slit openings are $100 \mu$ for entrance as well as exit slits and the sample size is $1 \mathrm{~mm} \times 1 \mathrm{~mm}$. 


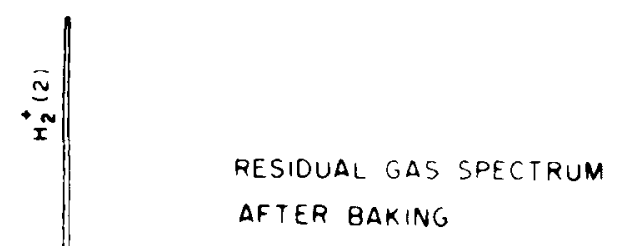

AFTER BAKING

Figure 9. Residual gas spectrum at ulti-

PRESSURE INSIOE THE CHAMBER $10 \times 10^{-9} \mathrm{~m}$ bor PRESSURE AT THE ANALYSER HEAD $35 \times 10^{-9} \mathrm{~m}$ bor mate pressure.

The authors acknowledge many useful discussions with Prof. M Watanabe, UVSOR facility, Japan regarding design aspects. The authors acknowledge with gratitude the technical help extended by the BARC and CAT workshops and the UHV group at CAT. Dr Saraswathy Padmanabhan and Ms Aparna Shastry's help during the course of work is acknowledged.

\section{References}

Das N C, Rajasekhar B N 1992 Design and evaluation of beamlines on synchrotron source. Proc. Int. Conf. on Synchrotron Radiation Sources, pp 350-354

Meenakshi Raja Rao P, Das N C, Rajasekhar B N, Kartha V B 1992 Design and development of photophysics beamline. Proc. Int. Conf. on Synchrotron Radiation Sources, Indore, pp 373-376

Meenakshi Raja Rao P, Rajasekhar B N, Saraswathy P, Das N C, Khan H A, Kartha V B, Raja Rao A S, Patel R J, Ratnakala K C, Sinha A K, Bhat S 1995 Mirror chambers for photophysics beamline at INDUS-I. Bull. Indian Vacuum Soc. 26: 7-15

Ramamurthi S S. 1992 Status of INDUS-I and INDUS-II. Proc. Int. Conf. on Synchrotron Radiation Sources, Centre for Advanced Technology, Indore, pp 9-16

Sakai K, Nakamura E, Yamazaki J, Matsudo O, Fukui K, Watanabe M 1987 Cooling of premirror at BLIB and BL7B. UVSOR activity report, pp 19-21 